\title{
SECAFÉ Parte II: Recomendaciones para el manejo eficiente de los secadores mecánicos de café pergamino
}

\author{
Alfonso Parra-Coronadoํ, Gonzalo Roa-Mejía ${ }^{2}$ \& Carlos E. Oliveros-Tascón ${ }^{2}$
}

\section{RESUMEN}

Una vez validados los modelos de simulación matemática para el secado de café pergamino (Modelos de Thompson y MSU) para los diferentes secadores existentes en Colombia, se procedió a implementar en lenguaje de programación Microsoft Visual Basic 6.0, un único programa de simulación, el cual fue utilizado para determinar las mejores condiciones de operar cada uno de los secadores mecánicos existentes y proporcionar recomendaciones para su manejo eficiente, con el fin de obtener café pergamino seco con contenido de humedad final entre 10 y $12 \%$ bh y predecir el tiempo de secado, la eficiencia térmica y el comportamiento del grano durante el proceso. Se realizaron en total 753 pruebas de simulación, lo cual corresponde a un promedio de 151 simulaciones por cada tipo de secador.

Palabras claves: simulación matemática, secado, modelo de Thompson, modelo MSU

\section{SECAFÉ Part II: Recommendations for the efficient operation of mechanical dryers for parchment coffee}

\begin{abstract}
A Microsoft Visual Basic 6.0 parchment coffee drying program was developed. Two separate programs, based on Thompson and MSU models and using Cenicafé's coffee parameters were successfully tested for the evaluation of the performance of all mechanical coffee dryers existing in Colombia. Based on the good results, a unique simulation program was implemented for drying parchment coffee and used to determine the most adequate ways to operate each one of the existing mechanical dryers and to give the necessary recommendations for their most efficient operation with the purpose of obtaining dry parchment coffee with final water content between 10 and $12 \% \mathrm{wb}$, and to predict the drying time, the thermal efficiency and the behavior of the grain during the drying. 753 tests of simulation were made altogether, which corresponds to an average of 151 simulations by each type of dryer.
\end{abstract}

Keywords: mathematical simulation, drying, Thompson's model, MSU model

1 Universidad Nacional de Colombia, Facultad de Ingeniería, Dpto. de Ingeniería Civil y Agrícola, Bogotá D. C., Colombia. Fone: (571) 3165430. Fax: (571) 3165462. E-mail:aparrac@unal.edu.co

2 Ingeniería Agrícola, Centro Nacional de Investigaciones de Café, Cenicafé. Chinchiná, Caldas, Colombia. Fone: (576) 850 6550. E-mail: gonzalo.roa@cafedecolombia.com, carlos.oliveros@cafedecolombia.com 


\section{INTRODUCCIÓN}

En el artículo "SECAFÉ Parte I: Implementación y validación de dos modelos de simulación matemática en el secado mecánico de café pergamino”, se presentó la forma como se desarrollaron, implementaron y validaron los programas de simulación matemática para el secado de café pergamino, tomando como base los modelos de Thompson y de la Universidad del Estado de Michigan (MSU). Además se planteó como principal tecnología (la más viable técnica y económicamente) el adecuado y oportuno proceso de secado de los granos, para lograr disminuir su contenido de humedad a niveles del $10-12 \%$ bh, que permitan su almacenamiento por periodos prolongados, incentivando al caficultor para que utilice técnicas de secado más eficientes, como es el caso del secado mecánico (convección forzada de aire), con los cuales se puede obtener ventajas como la reducción de la mano de obra, disminución del tiempo de secado y conservación de la calidad, de tal manera que permita obtener con menos riesgo "café tipo exportación” que cumpla con los requisitos de calidad exigidos por el mercado internacional.

En los sistemas de secado mecánico de café se logra reducir el contenido de humedad del grano desde 52-56\% bh, hasta el $10-12 \%$ bh, en un periodo de tiempo relativamente corto (18 a 45 h, dependiendo del tipo de secador y de las condiciones de operación del sistema), reduciendo así el riesgo de deterioro ocasionado por el ataque de hongos y de microorganismos. Sin embargo, si el secador no se opera adecuadamente, esto es, si a un determinado espesor de capa de grano no se le suministra la cantidad de aire necesaria a la temperatura adecuada, el secador no funcionará bien, pudiéndose comprometer la calidad del grano, o hacer que el consumo de energía aumente innecesariamente por operación inadecuada del ventilador y calentamiento de más aire del necesario, incrementando así los costos del proceso de secado (Parra, 2000).

Es importante operar los secadores de café con los correctos valores de caudal y temperatura del aire de secado. Los resultados de investigación de Cenicafé (Roa et al., 1999) indican que para secadores en capa estática el caudal mínimo recomendado es de $25 \mathrm{~m}^{3} \mathrm{~min}^{-1} \mathrm{~m}^{-3}$ de café pergamino (66 $\mathrm{m}^{3} \mathrm{~min}^{-1}$ por tonelada de café pergamino seco) y temperatura máxima del aire de secado de $50^{\circ} \mathrm{C}$.

Si se desea que los sistemas de secado de café, o de cualquier grano, sean eficientes, se debe conocer la manera de operarlos adecuadamente (espesor de la capa de grano, caudal y temperatura del aire más convenientes). El conocimiento de éstas condiciones de operación se pueden investigar por dos métodos diferentes, así:

1. Mediante estudios experimentales, lo cual es un procedimiento largo (de varios años), dispendioso y costoso (Alzate, 1992; Buitrago, 1991; Estevez \& Reyes, 1990; Loewer et al., 1994; Montenegro, 1992; Ospina, 1990; Rivera \& Vélez, 1997; Roa et al., 1999).

2. Mediante el desarrollo y aplicación de técnicas de simulación matemática del secado ejecutadas por computadores, por medio de los cuales se analizan las predicciones del comportamiento de los granos durante el proceso de secado y se efectúan las recomendaciones para el dimensionamiento y operación de los sistemas de secado. (Bakker-Arkema et al., 1974; Brooker et al., 1992; Cenkowski et al., 1993; Domínguez et al., 1983; Montoya, 1989; Parra, 1990; Parra, 1993; Roa et al., 1999; Thompson et al., 1968).

De acuerdo con lo anterior, se ha demostrado universalmente (Bakker-Arkema et al., 1974; Brooker et al., 1992; Parra, 1990; Rossi \& Roa, 1980; Thompson et al., 1968), que la simulación matemática del secado es una alternativa viable, tanto tecnológica como económicamente, razón por la cual se realizó esta investigación, con el propósito de generar conocimientos para la operación eficiente de los secadores mecánicos de café utilizados en Colombia.

En el artículo "SECAFÉ Parte I: Implementación y validación de dos modelos de simulación matemática en el secado mecánico de café pergamino”, se describen detalladamente los modelos de secado de Thompson (1968) y MSU (Michigan State University) desarrollado por Bakker-Arkema et al. (1974), los cuales fueron validados para los diferentes secadores de café pergamino existentes en Colombia. Las bondades de cada uno de ellos encontradas en el proceso de validación, permitió implementar un único programa de simulación (SECAFÉ), el cual fue utilizado para determinar las mejores condiciones de operar cada uno de los secadores mecánicos existentes y proporcionar recomendaciones para su manejo eficiente, con el fin de obtener café pergamino seco con contenido de humedad final entre $10 \mathrm{y}$ $12 \%$ bh y predecir el tiempo de secado, la eficiencia térmica y el comportamiento del grano durante el proceso.

Los secadores mecánicos utilizados en Colombia para el secado de café pergamino son de dos tipos (Roa et al., 1999): "secadores estáticos" y "secador de flujo continuo". Los secadores estáticos son: el secador con una sola cámara (secador de una sola capa) con inversión de la dirección del flujo de aire, el silo-secador "Cenicafé", el secador de dos pisos (secador de dos capas verticales) y el secador de tres pisos. El secador de flujo continuo es un "secador intermitente de flujos concurrentes, Cenicafé-IFC”, evaluado comercialmente en la Central de Beneficio del municipio de Anserma-Caldas. Con base en la manera como operan cada uno de estos secadores, se desarrollaron los programas de simulación matemática del secado para cada uno de ellos.

\section{MATERIAL Y METODOS}

Esta investigación se llevó a cabo en los laboratorios de Ingeniería Agrícola del Centro Nacional de Investigaciones de Café, Cenicafé, municipio de Chinchina - Caldas. Se desarrollaron las siguientes etapas investigativas: 1) obtención de los datos experimentales necesarios para las validaciones, 2) desarrollo de los programas de simulación, evaluación y aplicación de los modelos. En este artículo se presenta la metodología para la aplicación de los modelos de Thompson y MSU validados, los cuales se presentaron en el artículo "SECAFÉ Parte I: Implementación y validación de dos modelos de simulación matemática en el secado mecánico de café pergamino”. 
Aplicación de los programas de simulación desarrollados para secado de café pergamino.

Una vez validados los modelos de simulación para los diferentes secadores, se procedió a optimizarlos operacionalmente, con el fin de obtener café pergamino seco con contenido de humedad final entre 10 y $12 \%$ bh y predecir el tiempo de secado, la eficiencia térmica y el comportamiento del grano durante el proceso.

Teniendo en cuenta que el proceso de secado de granos se considera determinístico (Brooker et al., 1992) y utilizando como criterios de optimización: 1) mínimo tiempo de secado, 2) mínimo coeficiente de variación del contenido de humedad final del grano y 3) máxima capacidad dinámica del secador, se procedió a realizar diferentes combinaciones posibles de los parámetros de entrada (espesor de la capa de grano, caudal y temperatura del aire de secado) para seleccionar la(s) combinación(es) que presentara(n) el mínimo tiempo de secado y mínimo coeficiente de variación del contenido de humedad final del grano y máxima capacidad dinámica del secador. Se realizaron en total 753 pruebas de simulación, lo cual corresponde a un promedio de 151 simulaciones por cada secador. Los datos de entrada suministrados al programa de simulación seleccionado para la optimización de los secadores estáticos fueron:

\section{Datos del producto}

Contenido de humedad inicial_del café: 55,0\% bh

Temperatura inicial del café: $21,0^{\circ} \mathrm{C}$

Contenido de humedad promedio al final del secado: $11,0 \%$ bh

\section{Datos del lugar}

Altitud (Chinchiná, Caldas): 1.310,0 m.s.n.m.

Temperatura ambiente promedio: $21,0^{\circ} \mathrm{C}$.

\section{Datos del sistema}

Sección transversal del silo-secador: 1,0 $\mathrm{m}^{2}$.

Espesor de la capa de grano en cada cámara: Varió de 0,1 a $0,4 \mathrm{~m}$, con incrementos de $0,1 \mathrm{~m}$.

\section{Datos del aire de secado}

Temperatura: Se tomó como temperatura base del aire de secado, $50{ }^{\circ} \mathrm{C}$. Posteriormente se varió de 30 a $45^{\circ} \mathrm{C}$ con incrementos de $5^{\circ} \mathrm{C}$, con el fin de determinar el incremento de tiempo de secado al disminuir la temperatura con respecto a la temperatura base.

Humedad Relativa: Se tomó como base 0,17 (la correspondiente a una temperatura de $50{ }^{\circ} \mathrm{C}$, para la razón de humedad normal del lugar del experimento - Zona Cafetera Colombiana). Posteriormente se varió de acuerdo a la variación de la temperatura.

Caudal: Varió de 10 a $40 \mathrm{~m}^{3} \mathrm{~min}^{-1} \mathrm{~m}^{-2}$, con incrementos de $5 \mathrm{~m}^{3} \mathrm{~min}^{-1} \mathrm{~m}^{-2}$. Con base en los resultados obtenidos de la optimización, se procedió a elaborar las recomendaciones para operar de la manera más eficiente cada uno de los secadores utilizados para café pergamino, teniendo en cuenta una humedad media del $11 \%$ bh, con un coeficiente de variación del contenido de humedad final inferior a 6,5\% (lo que garantiza que la humedad final del grano esté entre el 10 y el $12 \%$ bh). Se tuvo en cuenta además que la mejor manera de operar el secador debería ser de fácil aplicación por parte del caficultor.

\section{RESULTADOS Y DISCUSIÓN}

Utilización del programa único de simulación desarrollado con base en los modelos de Thompson y de la Universidad Estatal de Michigan (MSU) para secado de café pergamino.

Con base en los análisis de validación de los modelos de Thompson y MSU, se procedió a implementar un único programa de simulación matemática de secado de café pergamino, conformado con las mejores opciones de los programas de Thompson, MSU y Thompson-MSU, para cada uno de los secadores estudiados. El programa unificado está conformado por 22 subrutinas para el secador intermitente de flujos concurrentes Cenicafé-IFC y 27 subrutinas para los secadores estáticos. En total se realizaron 753 simulaciones de secado para todas las condiciones de secado, distribuidas así:

- Para el secador estático de una sola cámara (sin cámaras de presecado), se realizaron 272 simulaciones.

- Para el silo secador "Cenicafé” y silo secador "Vertical” (dos pisos) se realizaron 212 simulaciones.

- Para el secador de tres pisos se realizaron 117 simulaciones.

- Para el secador intermitente de flujos concurrentes "Cenicafé-IFC”, se realizaron en total 152 simulaciones.

Para el "secador de tres pisos", las simulaciones se realizaron con los dos tipos de manejo, observándose que el "tipo de manejo simultáneo” presenta tiempos de secado similares al "tipo de manejo secuencial”; además, como era de esperarse, presenta mayores coeficientes de variación del contenido de humedad final del grano (menor uniformidad para las tandas 2 y 3) que el tipo de manejo secuencial y requiere de un mecanismo para la inversión del flujo de aire. Por estas razones, es más aconsejable el tipo de manejo secuencial.

Mediante la utilización del paquete de simulación, se logró establecer la mejor manera de operar cada uno de los secadores bajo condiciones específicas de secado. Se realizaron diagramas y tablas de operación para cada uno de los secadores utilizados en Colombia para café pergamino. Con ellos se puede determinar el tiempo de secado, tiempo adecuado para inversión del flujo de aire, capacidad dinámica del secador y eficiencia térmica global estimadas, con el fin de obtener café pergamino seco con contenidos de humedad entre 10 y $12 \%$ bh (estos contenidos de humedad se logran cuando los coeficientes de variación son inferiores a 6,5\%).

\section{Ejemplo de operación adecuada de secadores estáticos}

A continuación se indicará la manera de usar los diagramas obtenidos por simulación matemática para los diferentes tipos de secadores mecánicos utilizados para café pergamino, para lo cual se tomará como ejemplo la operación de un "silosecador Cenicafé”(o “silo-secador vertical”) con 0,4 m. de capa 
de café pergamino húmedo, tanto en la cámara de secado como dal de aire de $30 \mathrm{~m}^{3} \mathrm{~min}^{-1} \mathrm{~m}^{-2} \mathrm{y}$ la temperatura del aire de secado se mantiene en promedio en $50^{\circ} \mathrm{C}$.

Determinación del tiempo de secado: En las Figuras 1A y 1B se presentan los tiempos finales de secado en función del caudal de aire y del espesor de la capa de grano para el café ubicado en la cámara de secado y en la cámara de presecado. Ubicando en los diagramas la curva correspondiente a "espesor capa de grano =0,4 m." (localizada en la parte superior de los diagramas) y el valor del caudal de aire de $30 \mathrm{~m}^{3} \mathrm{~min}^{-1} \mathrm{~m}^{-2}$, se encuentran tiempos de secado de 23 y 32 h para el grano ubicado en la cámara de secado y en la cámara de presecado respectivamente (el grano en la cámara de presecado sale del secador 9 h después que el grano ubicado inicialmente en la cámara de secado).

Capacidad dinámica de secado y eficiencia térmica del secador (estimadas): En las Figuras 1C y 1D se presentan la capacidad dinámica de secado y la eficiencia térmica global del secador en función del caudal de aire y del espesor

A.

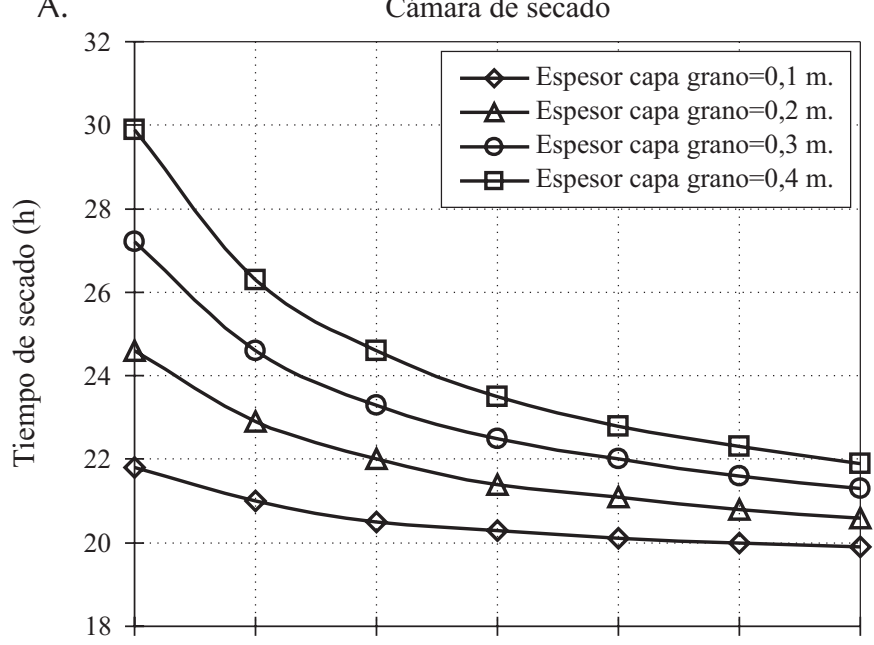

C.

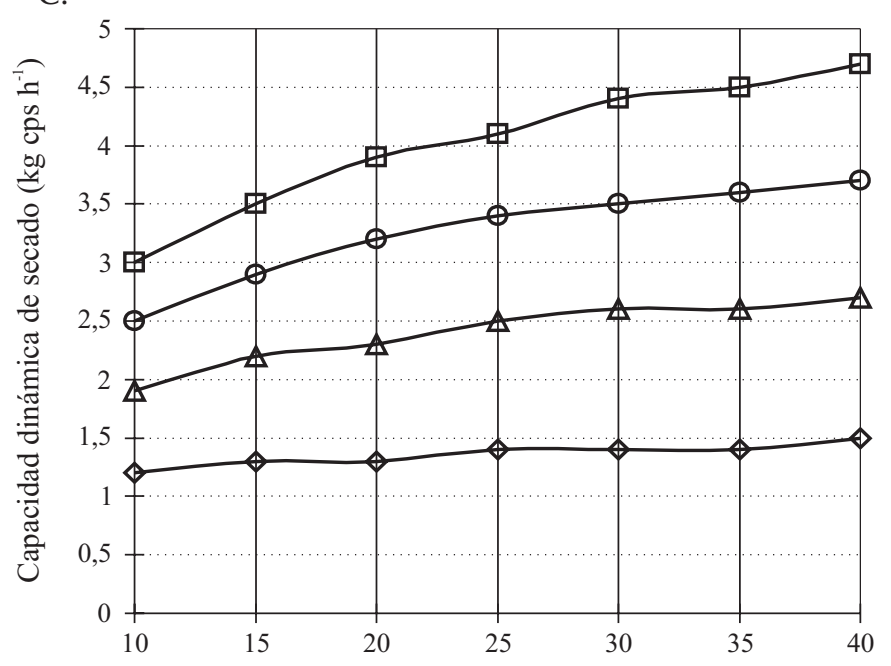
en la cámara de presecado. El ventilador suministra un cau-

de la capa de grano respectivamente, para el silo-secador "Cenicafé” y silo-secador vertical de dos pisos. La eficiencia térmica global del secador se expresa en \% y corresponde a la relación entre la energía necesaria para evaporar el agua del grano $\left(\mathrm{KJ} \mathrm{h}^{-1}\right)$ y la energía suministrada al aire que llega a la cámara de secado $\left(\mathrm{KJ} \mathrm{h}^{-1}\right)$.

Ubicando en los diagramas la curva correspondiente a “espesor capa de grano = 0,4 m" (localizada en la parte superior de los diagramas) y el valor del caudal de aire de $30 \mathrm{~m}^{3} \mathrm{~min}^{-1} \mathrm{~m}^{-2}$, se encuentra que el sistema de secado tiene una capacidad dinámica estimada de 4,4 kg de café pergamino seco (cps) $\mathrm{h}^{-1} \mathrm{~m}^{-2}$ y una eficiencia térmica global estimada del $22 \%$.

Intervalo de tiempo adecuado para la inversión del sentido del flujo de aire: En la Tabla 1 se presenta la información correspondiente a los intervalos de tiempo más adecuados para realizar la inversión del sentido del flujo de aire en este tipo de secador, de tal manera que se logre obtener un producto con buena uniformidad en el contenido de humedad final (dentro del rango 10 a $12 \%$ bh). De acuerdo con

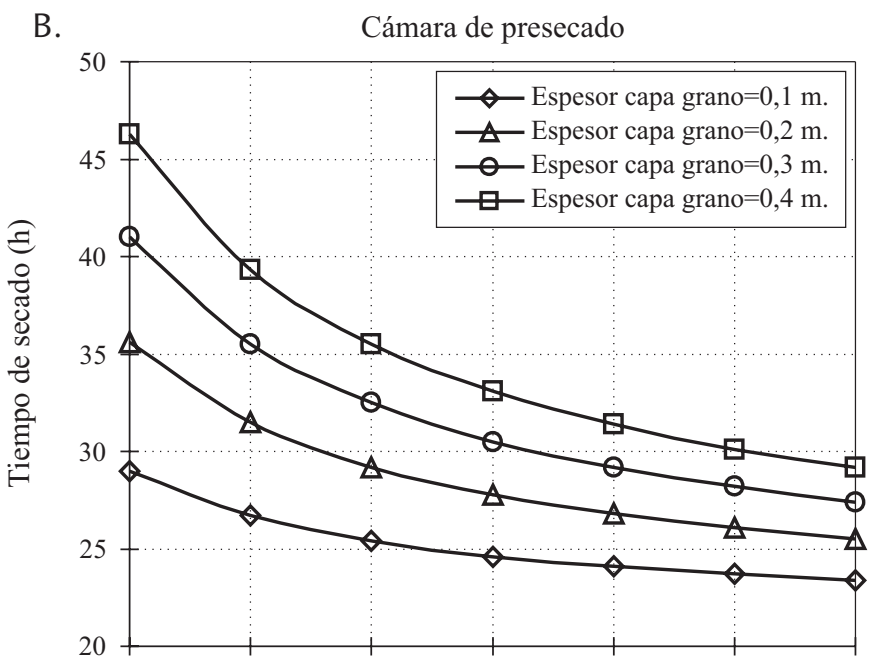

D.

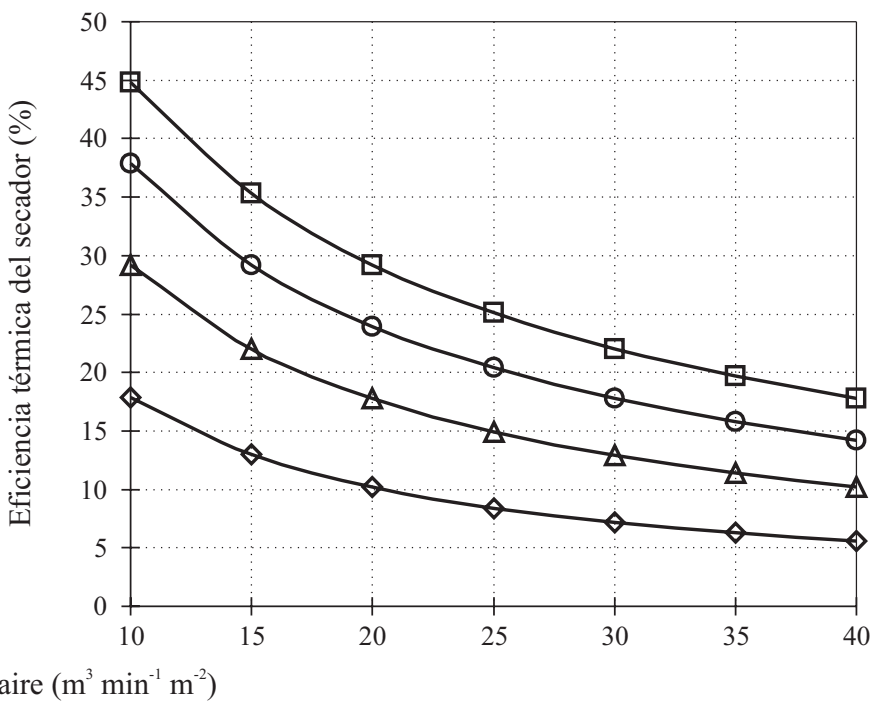

Figura 1. Silo-secador "Cenicafé" y silo-secador vertical (dos pisos). Diagramas de operación en función del caudal de aire y del espesor de la capa de grano para el café pergamino ubicado en las cámaras del secador. Temperatura del aire de secado: $50{ }^{\circ} \mathrm{C}$. 
esta tabla, para un caudal de aire de $30 \mathrm{~m}^{3} \mathrm{~min}^{-1} \mathrm{~m}^{-2}$ y un espesor de capa de grano de 0,4 m en cada una de las cámaras (secado y presecado), el intervalo de tiempo más adecuado para realizar la inversión del sentido del flujo de aire está entre 4 y $14 \mathrm{~h}$. Por lo tanto, se puede realizar la inversión del sentido del flujo de aire cada 8 h (o cada 10 o 12 h, según la conveniencia del operario). Se observa además, que a medida que aumenta el caudal de aire y disminuye el espesor de la capa de grano, los intervalos de tiempo para la inversión del sentido del flujo de aire se hacen más amplios, llegando a no requerirse inversión del flujo para altos caudales de aire y bajos espesores de capa de grano.

Tabla 1. Manejo óptimo del silo-secador "Cenicafé" y silo-secador vertical (dos pisos). Intervalos de tiempo (h) más adecuados para la inversión del sentido del flujo de aire

\begin{tabular}{ccccc}
\hline $\begin{array}{c}\text { Caudal de aire } \\
\left(\mathbf{m}^{3} \mathbf{~ m i n}^{-1} \mathbf{m}^{-2}\right)\end{array}$ & $\mathbf{5}$ & Espesor capa de grano $(\mathbf{m})$ \\
\cline { 2 - 5 } 10 & $6-9$ & $\mathbf{0 , 3}$ & $\mathbf{0 , 2}$ & $\mathbf{0 , 1}$ \\
\hline \multirow{2}{*}{10} & $4,8-9,7)^{\star}$ & $(3,3-7,5)$ & $(2,2-6,5)$ & $(0,7-4,2)$ \\
\hline \multirow{2}{*}{15} & $4-8$ & $4-12$ & $4-14$ & $4-16$ \\
& $(3,3-6,3)$ & $(2,2-6,6)$ & $(1,0-6,6)$ & $(0,1-5,2)$ \\
\hline \multirow{2}{*}{20} & $4-12$ & $4-14$ & $4-16$ & Sin Inversión \\
& $(2,1-6,6)$ & $(1,0-6,9)$ & $(0,8-7,2)$ & $(4,7-5,6)$ \\
\hline \multirow{2}{*}{25} & $4-14$ & $4-14$ & $4-16$ & Sin Inversión \\
& $(1,5-7,0)$ & $(0,8-6,4)$ & $(0,9-6,3)$ & $(3,9-4,5)$ \\
\hline \multirow{2}{*}{30} & $4-14$ & $4-16$ & $4-18$ & Sin Inversión \\
& $(0,9-6,7)$ & $(0,8-7,2)$ & $(0,3-6,2)$ & $(3,3-3,8)$ \\
\hline \multirow{2}{*}{35} & $4-14$ & $4-16$ & Sin Inversión & Sin Inversión \\
& $(0,7-6,3)$ & $(0,9-6,6)$ & $(5,7-6,7)$ & $(2,9-3,3)$ \\
\hline \multirow{2}{*}{40} & $4-16$ & $4-18$ & Sin Inversión & Sin Inversión \\
& $(0,8-7,3)$ & $(0,4-6,8)$ & $(5,1-5,9)$ & $(2,5-2,9)$ \\
\hline
\end{tabular}

* Coeficiente de variación del contenido de humedad final en \%; Temperatura del aire de secado $=50^{\circ} \mathrm{C}$.

Notas:

1. El Silo-secador vertical (dos pisos), opera con ésta misma tabla para la cámara de secado (Tanda 1). Al pasar el grano que se encuentra en la cámara de presecado a la cámara de secado, se debe invertir las capas de grano y no realizar inversión del flujo de aire posteriormente.

2. A menor tiempo de inversión del flujo de aire, menor es el coeficiente de variación del contenido de humedad final (mayor uniformidad en el contenido de humedad final del grano). Para coeficientes de variación menores a 7,3\%, el contenido de humedad final del grano se encuentra en el rango de 10 a $12 \%$ bh.

Incremento del tiempo de secado cuando la temperatura del aire es inferior a $50^{\circ} \mathrm{C}$ : Si en el mismo ejemplo anterior se supone que la temperatura del aire de secado es de 40 y no de $50{ }^{\circ} \mathrm{C}$, se obtiene una mayor uniformidad en el contenido de humedad final del grano (disminuye el coeficiente de variación), pero aumenta el tiempo de secado. El diagrama de la Figura 2 permite determinar en cuanto se incrementa el tiempo de secado (para los granos en las cámaras de secado y de presecado), cuando la temperatura del aire de secado es inferior a $50^{\circ} \mathrm{C}$. De acuerdo con el diagrama, para una temperatura de $40^{\circ} \mathrm{C}$ el tiempo de secado se incrementa en 17 y 20 h para las cámaras de secado y de presecado respectivamente. En otros términos, si el tiempo de secado a $50{ }^{\circ} \mathrm{C}$ es de 23 y $32 \mathrm{~h}$ para el grano ubicado en las cámaras de secado y de presecado respectivamente (se obtienen de las Figuras 1A y 1B), estos se incrementarán a 40 y 52 h cuando se utilice una temperatura del aire de secado de $40{ }^{\circ} \mathrm{C}$.

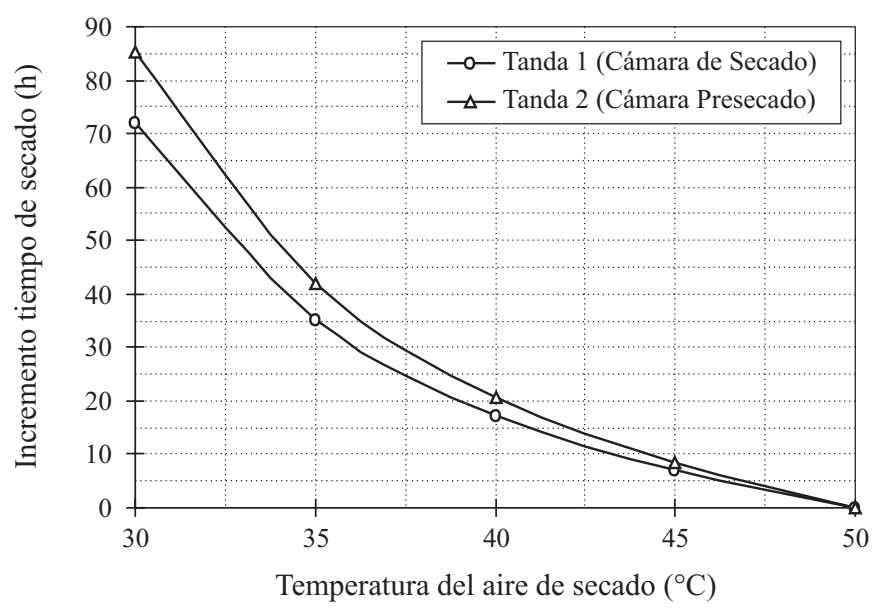

Figura 2. Silo-secador "Cenicafé" y silo-secador vertical (dos pisos). Incremento del tiempo de secado en función de la temperatura del aire, cuando ésta es inferior a $50{ }^{\circ} \mathrm{C}$.

Los resultados obtenidos mediante el programa único de simulación, presentan las siguientes tendencias generales para los diferentes secadores estáticos utilizados para café pergamino:

1. A medida que aumenta el caudal de aire y disminuye el espesor de la capa de grano, los tiempos de secado se hacen menores (Figura 1A y 1B), el contenido de humedad final del grano es más uniforme (disminuye el Coeficiente de Variación), los tiempos óptimos de inversión del flujo de aire se hacen menores (Tabla 1) y la eficiencia térmica global del secado disminuye (aprovechamiento menos eficiente de la energía utilizada para el secado) (Figura 1D).

2. La Capacidad Dinámica de secado aumenta a medida que aumenta el caudal de aire y el espesor de la capa de grano en cada cámara del secador, lo cual es más notorio para espesores de capa superiores a 0,2 m (Figura 1C). Por esta razón, no es muy aconsejable operar los secadores estáticos con espesores de capa de grano inferiores a 0,2 m.

De igual manera, en las simulaciones realizadas se observan las siguientes características para los secadores intermitentes de flujos concurrentes "Cenicafé-IFC":

1. Cuando se realizaron simulaciones con un espesor de la cámara de secado superior a 0,65 m, se observó que ocurrían variaciones mínimas en el contenido de humedad y temperatura final del grano, así como en el tiempo total de secado. Este mismo comportamiento fue observado por Montoya (1989), para una altura de la columna de secado de $0,6 \mathrm{~m}$, quien sugirió un espesor adecuado de columna de secado de $0,65 \mathrm{~m}$. Se recomienda usar la mínima altura (0,65 m) para disminuir los requerimientos de potencia del ventilador. 
2. Las simulaciones mostraron que existen diferentes combinaciones de flujo de aire (caudal) y temperatura del aire de secado, bajo las cuales el secador opera eficientemente, para entregar el grano con una temperatura inferior o igual a $50{ }^{\circ} \mathrm{C}$ al final del secado. Para las diferentes combinaciones de flujo y temperatura del aire de secado, se obtuvieron diferencias máximas absolutas de $4 \mathrm{~h}$ en los tiempos totales de secado. De acuerdo con los resultados obtenidos con el modelo de simulación, se puede utilizar cualquiera de las combinaciones de flujo y temperatura del aire de secado, con el fin de obtener café pergamino seco con temperaturas inferiores a $50{ }^{\circ} \mathrm{C}$ y contenidos finales de humedad entre 10 y $12 \%$ bh.

3. De las combinaciones de flujo y temperatura del aire de secado que se consideran adecuadas para dimensionar y operar un secador intermitente de flujos concurrentes “Cenicafé-IFC", la que arroja mejores resultados al variar los flujos de café húmedo a través del secador, es la correspondiente a una temperatura del aire de secado de $80^{\circ} \mathrm{C}$ y un flujo de aire de $50 \mathrm{~m}^{3} \mathrm{~min}^{-1} \mathrm{~m}^{-2}$, obteniéndose las siguientes condiciones para la operación del secador, cuando el contenido de humedad del café se reduce de 54 a $10,5 \%$ bh:

- Temperatura del aire de secado: $80{ }^{\circ} \mathrm{C}$

- Flujo de aire: $50 \mathrm{~m}^{3} \mathrm{~min}^{-1} \mathrm{~m}^{-2}$

- Flujo de café pergamino: $800 \mathrm{~m}^{3} \mathrm{~h}^{-1} \mathrm{~m}^{-2}$

- Longitud de la columna de secado: 0,65 m

- Longitud sección de reposo (para Índice de redistribución de 0,75$)^{1}: 1,2 \mathrm{~m}$

- Tiempo estimado de secado 2 : 23,5 h

- Número de vueltas (número de veces que el grano pasa por la sección de secado): 14

\section{CONCLUSIONES}

1. Con los diagramas y tablas de operación para cada uno de los secadores mecánicos existentes para café pergamino, obtenidos mediante los programas de simulación implementados, es posible determinar las mejores condiciones de operación de cada secador, de acuerdo con las condiciones específicas de secado. Con ellos se puede determinar el tiempo de secado, el tiempo adecuado para inversión del flujo de aire, la capacidad dinámica del secador y la eficiencia térmica global estimadas, con el fin de obtener café pergamino seco con contenidos de humedad entre 10 y $12 \%$ bh.

2. De acuerdo con las simulaciones realizadas, no es aconsejable operar los secadores estáticos con espesores de capa de grano inferiores a $0,2 \mathrm{~m}$ cuando se utilizan caudales de aire superiores a $20 \mathrm{~m}^{3} \mathrm{~min}^{-1} \mathrm{~m}^{-2}$, ya que bajo estas condiciones, la capacidad dinámica de secado y la eficiencia térmica del secador son muy bajas.

3. Los diagramas realizados para los secadores intermitentes de flujos concurrentes “Cenicafé-IFC”, además de su- ministrar información para la operación adecuada del secador, permiten dimensionarlo, especialmente en lo relacionado con la longitud de la sección de reposo, la cual aumenta a medida que aumenta el índice de redistribución de humedad y el flujo de café. La longitud más adecuada de la cámara de secado es de $0,65 \mathrm{~m}$, con la cual se obtienen los menores requerimientos de potencia del ventilador. El número de vueltas (veces que el grano pasa por la sección de secado) aumenta al aumentar el flujo de café.

\section{LITERATURA CITADA}

Alzate, J. G. Optimización operacional del silo-secador CENICAFE. Medellín: Departamento de Ingeniería Agrícola/Universidad Nacional de Colombia. 1992. 240p. Tesis Ingeniero

Bakker-Arkema, F. W.; Lerew, L. E.; De Boer, S. F.; Roth, M. C. Grain drying simulation. Michigan: Research report from de Michigan State University East Lansing-MI. 1974. 80p.

Brooker, D. B.; Bakker-Arkema, F. W.; Hall, C. W. Drying and storage of grains and oilseeds. An AVI book, New York: Published by Van Nostrand Reinhold. 1992. 450p.

Buitrago, O. Implementación del secado mecánico de café en carros secadores. Bogotá: Departamento de Ingeniería Agrícola/Universidad Nacional de Colombia. 1991. 184p. Tesis Ingeniero

Cenkowski, S.; Jayas, D. S.; Pabis, S. Deep-Bed Grain Drying A Review of particular theories. Drying Technology, v.11, n.7, p.1553-1581, 1993.

Dominguez, J. Parra, A.; Villa, L. G. Simulación matemática y optimización del secado de productos agropecuarios con aire natural y energía solar. Revista Ingeniería e Investigación, v.2, n.3, p.57-62, 1983.

Estevez, J. P.; Reyes, V. R. Secador por deshumidificación de aire e inversión de flujo, diseño y evaluación. Bucaramanga: Facultad de Ingeniería Mecánica/Universidad Industrial de Santander, 1990. 255p. Proyecto Grado

Loewer, O. J.; Bridges, T. C.; Bucklin, R. A. On farm drying and storage systems. American Society of Agricultural Engineers, Pam DeVore-Hansen Acquisitions; Books \& Journals, St. Joseph: ASAE, 1994. 560p. Publication 9

Montenegro, J. Y. Secador eléctrico estático para café de baja capacidad, inversión de flujo y recirculación de aire. Bogotá: Departamento de Ingeniería Agrícola/ Universidad Nacional de Colombia, 1992, 161p. Tesis Ingeniero

Montoya, E. C. Optimización operacional del secador intermitente de flujos concurrentes para café pergamino. Pereira: Facultad de Ingeniería Industrial/Universidad Tecnológica de Pereira, 1989. 116p. Tesis MSc

Ospina, J. E. Silo Secador CENICAFE. Informe de año sabático. Federación Nacional de Cafeteros de Colombia. Chinchiná: Centro Nacional de Investigaciones de Café "Pedro Uribe Mejía”, 1990. 57p.

\footnotetext{
${ }^{1}$ La longitud de la sección de reposo varía con el índice de redistribución de la humedad en el grano y con el flujo de café pergamino a través del secador.

2 Es el tiempo requerido para llevar el café pergamino de un contenido de humedad de 54 a 10,5\% bh
} 
Parra, A. Potencial de secado de yuca con aire natural y energía solar en las regiones productoras de Colombia. Revista latinoamericana ACOGRANOS, año 6, n.8, p.1217, 1990.

Parra, A. Almacenamiento aireado de maíz en los Llanos Orientales. Revista Ingeniería e Investigación, v.8, n.29. p.5-13, 1993.

Parra, A. Evaluación y utilización práctica de los modelos de simulación matemática de Michigan y de Thompson en el secado de café con aire forzado. Chinchiná - Caldas: CENICAFÉ, 2000. 108p. Experimento ING-0824, Informe final.
Rivera, O. L.; Velez P. A. Evaluación de una secadora de café de 12 arrobas (para pequeños cafeteros). Medellín: Corporación Universitaria Lasallista, Ingeniería de alimentos, 166p, 1997. Trabajo Grado

Roa, G.; Oliveros, C. E.; Álvarez, J.; Ramírez, C.; Sanz, J. R.; Dávila, M. T.; Álvarez, J. R.; Zambrano, D. A.; Puerta, G. I.; Rodríguez, N. Beneficio ecológico del café. Chinchiná: CENICAFE, 1999, 300p.

Rossi, J. R.; Roa, G. Secagem e armazanamento de produtos agropecuarios com uso de energia solar e ar natural. Sao Pablo: ACIESP, 1980, 293p.

Thompson, T. L. Peart, R. M.; Foster, G. H. Mathematical simulation of corn drying - A new model. Transaction of the ASAE, v.11, n.4, p.582-586, 1968. 